\title{
Noninvasive Brain Stimulation and Neural Entrainment Enhance Athletic Performance—a Review
}

\author{
Lorenza S. Colzato $^{1}$ - Michael A. Nitsche $e^{2,3,4} \cdot$ Armin Kibele $^{5}$
}

Received: 25 May 2016 / Accepted: 13 October 2016/Published online: 31 October 2016

(C) The Author(s) 2016. This article is published with open access at Springerlink.com

\begin{abstract}
Brain stimulation and neural entrainment relying on noninvasive techniques, applied to sports, might enhance brain activity in healthy athletes to improve their physical performance. In the past, several studies have employed stimulation procedures, either during athletic training or during separate sessions, to enhance physical and mental performance. Here, we review the available physiological and behavioral studies to clarify if and under which conditions noninvasive brain stimulation and neural entrainment might enhance athletic performance. Even though many studies suffer from small sample size, the results, compared to traditional training procedures, suggest advantages with regard to motor learning, motion perception, muscular strength, or decrements in muscle fatigue. Further, these techniques seem to be useful in fine-tuning crucial aspects of competitive sports such as speeding up the learning rate of specific motor skills. Although more research is needed to fully understand the effects of noninvasive brain stimulation and neural entrainment on athletic performance, we conclude that these
\end{abstract}

Lorenza S. Colzato

colzato@fsw.leidenuniv.nl

1 Cognitive Psychology Unit \& Leiden Institute for Brain and Cognition, Leiden University, Wassenaarseweg 52, 2333

AK Leiden, The Netherlands

2 Department of Clinical Neurophysiology, Georg-August University Göttingen, Göttingen, Germany

3 Leibniz Research Centre for Working Environment and Human Resources, Dortmund, Germany

4 Department of Neurology, University Medical Hospital Bergmannsheil, Bochum, Germany

5 Institute for Sports and Sport Science, University of Kassel, Kassel, Germany emerging techniques are promising tools to enhance physical and mental performances in sports.

Keywords Noninvasive brain stimulation $\cdot$ Neural entrainment $\cdot$ Sport $\cdot$ Performance $\cdot$ Enhancement

\section{Introduction}

Citius, altius, fortius - faster, higher, stronger - these three adjectives incorporate the spirit of competitive sport, not only for professional but also for amateur athletes. The huge pressure exerted by trainers on athletes to enhance performance and "to be on top" all the time has raised the interest in boosting performance using new methods and materials. In recent years, the application of nonpharmacological brain stimulation and neural entrainment techniques in sports science has become increasingly popular as a topic of scientific research because of their suggested enhancing effects on athletic performance (see, Grosprêtre et al. 2016, for a recent review on transcranial magnetic stimulation on sport). Conceptually, noninvasive brain stimulation techniques, such as transcranial direct current stimulation (tDCS), and neural entrainment techniques, such as neurofeedback and transcranial alternating current stimulation (tACS), operate on different mechanisms of action. Whereas tDCS modifies brain excitability through weak, direct electric currents, neurofeedback and tACS are techniques influencing the brain control on human action by modulation of brain oscillations. Recently, Davis (2013) has coined the term "neurodoping" to indicate the use of these emerging techniques to enhance physical and mental performance in sports. Indeed, factors that are considered crucial to athletic performance, such as motor learning, enhanced muscular strength or reduced fatigue, or even speeding up the learning rate of specific motor skills can be promoted by the 
use of brain stimulation and neural entrainment techniques (Table 1).

In this review, we will first describe the available studies investigating how noninvasive brain stimulation (tDCS) can increase athletic performance. Second, we will outline studies exploring the beneficial effect of neural entrainment (tACS and neurofeedback) on various sports. The studies indicate that these emerging techniques are promising tools to enhance physical and mental performances in sports.

\section{Improvements in Muscular Strength, Motion Perception, Motor Learning, and Muscle Fatigue Through Brain Stimulation}

The effects of noninvasive brain stimulation procedures, such as tDCS, on athletic performance have been investigated in several studies. tDCS delivers a low-intensity constant current, usually between 1 and $2 \mathrm{~mA}$, via electrodes that are applied on the participant's scalp above brain regions of interest for a variable amount of time (usually for 5 to $20 \mathrm{~min}$ ). A portion of the applied current penetrates the brain and is effective in altering spontaneous neural activity and excitability (Nitsche et al. 2008). The current applied to the brain through tDCS is not sufficiently strong to generate action potentials (Nitsche et al. 2008). Indeed, tDCS induces a sub-threshold modulation of the resting membrane potential of cortical neurons, changing their likelihood of firing and consequently impacting spontaneous cortical activity (Nitsche and Paulus 2000; Nitsche et al. 2003a, 2008). The tDCS-induced changes in the resting membrane potential are for the most part regulated by the polarity of the stimulation. Anodal stimulation induces a slight depolarization of the resting membrane potential, most likely at the soma and axon of the targeted neurons, which raises the probability of neural firing and, accordingly, cortical excitability (Nitsche and Paulus 2000). In contrast, cathodal stimulation causes a slight hyperpolarization of the resting membrane potential of respective structures and thereby reduces the probability of neural firing and excitability (Nitsche and Paulus 2000). Shifts in neural activity take place during the stimulation period as well as after the stimulation period, if the current is delivered for a sufficient period of time (i.e., at least 9-10 $\mathrm{min}$ ). Such shifts can last for longer than $1 \mathrm{~h}$ after the stimulation has ended and are assumed to resemble plasticity of glutamatergic synapses (Nitsche and Paulus 2000; Nitsche et al. 2003a, 2008).

In this section, we will give an overview of recent findings of tDCS on muscular strength, motion perception, motor learning, and muscle fatigue. Cogiamanian et al. (2007) examined the effects of tDCS on neuromuscular fatigue in submaximal isometric contractions of the left elbow in 24 healthy participants (who did not participate in competitive sports). The authors found a significant $15 \%$ decrease in the neuromuscular fatigue when anodal tDCS was applied over the motor areas of the scalp. This outcome suggests that anodal tDCS can improve muscle performance and decrease muscle fatigue. Additional evidence for an enhancement of muscle endurance comes from a study in which sustained submaximal contractions of the elbow flexion were examined in 18 healthy participants (Williams et al. 2013). During fatigue task performance, either anodal or sham stimulation was administered to the motor cortex for up to $20 \mathrm{~min}$.

The results indicate that anodal stimulation increased time to task failure and the amount of muscle fatigue, suggesting that the administration of tDCS during performance of fatiguing activity is able to augment the capability to exercise under challenging conditions (Williams et al. 2013). Further, a recent study investigated the effect of anodal tDCS on perceived exertion, heart rate, and performance outcome during a maximal incremental cycling protocol in ten experienced male cyclists (Okano et al. 2013). Anodal tDCS was applied over the left temporal cortex because this brain area is associated with autonomic nervous system control and previous evidence has shown that its stimulation was successful in modulating this system (Montenegro et al. 2011). The stimulation resulted in a slower increase of perceived exertion during exercise. Moreover, peak power output (the highest intensity a cyclist can sustain for more than $1 \mathrm{~min}$ ) was increased by $4 \%$, with heart rates reduced at submaximal workloads (Okano et al. 2013). Similarly, Vitor-Costa et al. (2015) investigated the enhancing effect of tDCS over the primary motor cortex on muscle fatigue and exercise tolerance in 11 cyclists. Anodal tDCS enhanced the time to exhaustion at $80 \%$ of peak power output. However, no significant effects were found on perceived exertion and heart rate, indicating that anodal tDCS selectively enhanced performance without affecting physiological and perceptual variables.

Aside from the beneficial effect on muscle fatigue, a recent study has shown an enhancing effect of tDCS on implicit motor learning through a stimulation procedure over the left dorsal-lateral prefrontal cortex (Zhu et al. 2015). Here, cathodal tDCS and sham stimulation were compared in 27 healthy participants, showing improvements in golf putting while verbal working memory performance was impaired by this stimulation procedure. The authors suggest that the suppression of verbal working memory induced by cathodal tDCS induced impairments in explicit control strategies and consequently promoted implicit motor learning of the golf putting skill. Earlier, the effects of noninvasive cortical stimulation were studied by Nitsche et al. (2003b): the primary motor cortex, premotor, or prefrontal cortices were stimulated contralaterally to the performing hand during performance of a serial reaction time task. Anodal stimulation of the primary motor cortex increased performance, while stimulation of the remaining cortices had no effect. Accordingly, the authors suggested that the primary motor cortex is involved in the 
Table 1 Overview of studies including technique used to enhance sport performance or sport related functions

\begin{tabular}{|c|c|c|c|c|c|}
\hline Study & Sample & Technique & Sport activity & $\begin{array}{l}\text { Target brain } \\
\text { area }\end{array}$ & $\begin{array}{l}\text { Skill/ } \\
\text { performance } \\
\text { affected }\end{array}$ \\
\hline $\begin{array}{c}\text { Antal et al. } \\
\text { (2004a) }\end{array}$ & $N=42$ & Anodal tDCS & Nonathletes & $\begin{array}{l}\text { Middle } \\
\quad \text { temporal } \\
\quad(\mathrm{MT}+\mathrm{V} 5)\end{array}$ & $\begin{array}{l}\uparrow \text { visuo-motor } \\
\text { coordination }\end{array}$ \\
\hline $\begin{array}{c}\text { Antal et al. } \\
\text { (2004b) }\end{array}$ & $N=12$ & Cathodal tDCS & Nonathletes & Visual cortex & $\uparrow \underset{\text { perception }}{\text { motion }}$ \\
\hline $\begin{array}{l}\text { Arns et al. } \\
\quad(2008)\end{array}$ & $N=6$ & $\begin{array}{l}\text { Neurofeedback } \\
\text { Personalized EEG profile }\end{array}$ & $\begin{array}{l}\text { Amateur } \\
\text { golfers }\end{array}$ & $\begin{array}{l}\text { Frontopolar } \\
\text { cortex }\end{array}$ & $\begin{array}{l}\uparrow \text { golf putting } \\
\text { performance }\end{array}$ \\
\hline $\begin{array}{l}\text { Cogiamanian } \\
\text { et al. } \\
(2007)\end{array}$ & $N=24$ & Anodal tDCS & Nonathletes & Motor cortex & $\begin{array}{c}\downarrow \text { muscular } \\
\text { fatigue }\end{array}$ \\
\hline $\begin{array}{l}\text { Kao et al. } \\
\qquad(2014)\end{array}$ & $N=3$ & $\begin{array}{l}\text { Neurofeedback } \\
\text { Reduced frontal midline } \\
\text { theta }\end{array}$ & Skilled golfers & $\begin{array}{l}\text { Medial } \\
\text { frontal } \\
\text { cortex }\end{array}$ & $\begin{array}{l}\uparrow \text { golf putting } \\
\text { performance }\end{array}$ \\
\hline $\begin{array}{l}\text { Landers et al. } \\
\text { (1991) }\end{array}$ & $N=24$ & $\begin{array}{l}\text { Neurofeedback } \\
\text { Enhanced low frequency } \\
\text { activity }\end{array}$ & $\begin{array}{l}\text { Pre-elite } \\
\text { archers }\end{array}$ & $\begin{array}{l}\text { Left temporal } \\
\text { cortex }\end{array}$ & $\begin{array}{l}\uparrow \text { arrow } \\
\text { shooting } \\
\text { performance }\end{array}$ \\
\hline $\begin{array}{l}\text { Nitsche et al. } \\
\text { (2003b) }\end{array}$ & $N=80$ & Anodal tDCS & Nonathletes & $\begin{array}{l}\text { Primary } \\
\text { motor } \\
\text { cortex }\end{array}$ & $\begin{array}{l}\uparrow \text { implicit motor } \\
\text { learning }\end{array}$ \\
\hline $\begin{array}{l}\text { Okano et al. } \\
\text { (2013) }\end{array}$ & $N=10$ & Anodal tDCS & $\begin{array}{l}\text { Experienced } \\
\text { male } \\
\text { cyclists }\end{array}$ & $\begin{array}{l}\text { Left temporal } \\
\text { cortex }\end{array}$ & $\begin{array}{l}\downarrow \text { perceived } \\
\quad \text { exertion } \\
\text { during } \\
\text { exercise }\end{array}$ \\
\hline $\begin{array}{l}\text { Pollok et al. } \\
\text { (2015) }\end{array}$ & $N=26$ & Alpha and beta tACS & Nonathletes & $\begin{array}{l}\text { Left primary } \\
\text { motor } \\
\text { cortex }\end{array}$ & $\begin{array}{c}\uparrow \text { sequence } \\
\text { learning }\end{array}$ \\
\hline $\begin{array}{l}\text { Reis et al. } \\
\text { (2009) }\end{array}$ & $N=24$ & $\begin{array}{r}\text { Anodal tDCS for } 5 \\
\text { consecutive days }\end{array}$ & Nonathletes & $\begin{array}{l}\text { Primary } \\
\text { motor } \\
\text { cortex }\end{array}$ & $\begin{array}{l}\uparrow \text { learning } \\
\text { challenging } \\
\text { motor skill }\end{array}$ \\
\hline $\begin{array}{l}\text { Ring et al. } \\
\text { (2015) }\end{array}$ & $N=24$ & $\begin{array}{l}\text { Reduced alpha power } \\
\text { neurofeedback }\end{array}$ & Golfers & $\begin{array}{l}\text { Frontal site } \\
\quad(\mathrm{Fz})\end{array}$ & $\begin{array}{l}\text { No } \\
\text { improvement } \\
\text { in golf } \\
\text { putting } \\
\text { performance }\end{array}$ \\
\hline $\begin{array}{l}\text { Rostami et al. } \\
\text { (2012) }\end{array}$ & $N=24$ & $\begin{array}{l}\text { Intermediate alpha power } \\
\text { and reduced theta and } \\
\text { high beta } \\
\text { neurofeedback }\end{array}$ & $\begin{array}{r}\text { Expert rifle } \\
\text { shooters }\end{array}$ & $\begin{array}{l}\text { Parietal site } \\
\quad(\mathrm{Pz})\end{array}$ & $\begin{array}{l}\uparrow \text { shooting } \\
\text { performance }\end{array}$ \\
\hline $\begin{array}{l}\text { Vitor-Costa } \\
\quad \text { et al. } \\
\quad(2015)\end{array}$ & $N=11$ & Anodal tDCS & Cyclists & $\begin{array}{l}\text { Primary } \\
\text { motor } \\
\text { cortex }\end{array}$ & $\begin{array}{l}\uparrow \text { time to } \\
\text { exhaustion }\end{array}$ \\
\hline $\begin{array}{l}\text { Williams } \\
\text { et al. } \\
\text { (2013) }\end{array}$ & $N=18$ & Anodal tDCS & Nonathletes & Motor cortex & $\begin{array}{l}\uparrow \text { muscle } \\
\text { endurance }\end{array}$ \\
\hline $\begin{array}{l}\text { Zhu et al. } \\
\text { (2015) }\end{array}$ & $N=27$ & Cathodal tDCS & $\begin{array}{l}\text { Healthy } \\
\text { students } \\
\text { with no golf } \\
\text { experience }\end{array}$ & $\begin{array}{l}\text { Left } \\
\text { dorsal-- } \\
\text { lateral } \\
\text { prefrontal } \\
\text { cortex }\end{array}$ & $\begin{array}{l}\uparrow \text { golf putting } \\
\text { performance }\end{array}$ \\
\hline
\end{tabular}

$\downarrow$ decreased, $\uparrow$ increased, $t A C S$ transcranial alternating current stimulation, $t D C S$ transcranial direct current stimulation acquisition and early consolidation phase of implicit motor learning. Along the same lines, another study investigated the effect of tDCS on the extended time course of learning a novel and challenging motor skill task (Reis et al. 2009). Two groups of 12 subjects practiced over five consecutive days while receiving tDCS over the primary motor cortex. Within-day and between-day effects and on the rate of forgetting during a 3-month follow-up were examined. There was 
greater total skill acquisition with anodal tDCS compared to sham. Anodal tDCS did not change the rate of forgetting relative to sham across the 3-month follow-up period, but the skill measure remained greater at 3 months.

Finally, two studies investigated the effects of tDCS on performance on visuo-motor tasks. First, Antal et al. (2004a) examined whether the human middle temporal (MT)+/V5, an extrastriate visual area that is known to mediate motion processing, and the primary motor cortex are implicated in learning of visuo-motor coordination (as indexed by a visually guided tracking task). The percentage of correct tracking movements increased significantly in the early learning phase during anodal stimulation, but only when the left V5 or M1 was stimulated. In contrast, cathodal stimulation showed no significant effect. Second, tDCS of the visual cortex was found to improve motion perception in a visuo-motor tracking task (Antal et al. 2004b). Here, 12 participants received cathodal and anodal stimulation over different areas in the visual cortex and the motor cortex. The percentage of correct tracking movements increased specifically during and immediately after cathodal stimulation of V5, area responsible for motion perception only, while no effects were found for the other stimulation conditions.

In sum, from the above studies, tDCS seems to be a promising tool to enhance muscular strength, motion perception, motor learning, and fatigue, which are all crucial for athletic performance. However, more research is needed for unequivocal support for this idea. So far, optimal protocols of stimulation (stimulation duration, intensity, target and return electrode positions) are yet to be identified. Moreover, it needs to be clarified whether tDCS might be useful in highly trained individuals. Indeed, maximum performance in fine motor control could not be further improved in elite pianists (Furuya et al. 2013). Accordingly, it needs to be investigated whether similar ceiling effects might apply to the performance of elite athletes as well.

\section{Improvements in Motor Sequence Learning and Motor Skill Learning Through Neural Entrainment Procedures}

In this section, studies will be reviewed investigating the effects of neural entrainment procedures on athletic performance including tACS and neurofeedback. The idea behind neural entrainment is that the rhythmic oscillatory activity within and between different brain regions is considered to play a causal role in a wide range of cognitive functioning and can be modulated by respective interventions.

Similar to tDCS, tACS protocols apply weak electrical current to the scalp through two or more electrodes placed over brain areas of interest. However, while tDCS is used to induce a constant current flow, tACS is used to apply an oscillatory (sinusoidal) electrical stimulation of a specific frequency to modulate neuronal membrane potentials in a frequencydependent manner. tACS is usually applied with stimulation intensities and durations comparable to tDCS and at oscillation frequencies within the EEG frequency spectrum (typically between 1 and $100 \mathrm{~Hz}$ ). When applied within the conventional EEG frequency spectrum, tACS does not seem to induce neuroplasticity but instead its primary effect seems to modulate the spontaneous ongoing cortical oscillations (Antal et al. 2008). Particularly, tACS is supposed to enhance cortical oscillations at frequencies close to the stimulation frequency and to entrain or synchronize neuronal networks (Reato et al. 2010, 2013). However, when tACS is applied outside the typical EEG frequency range (e.g., at 140, $600 \mathrm{~Hz}$, and in the low $\mathrm{kHz}$ range), tDCS-like neuroplastic excitability alterations are more likely to occur (Moliadze et al. 2010, 2012; Chaieb et al. 2011). Recently, Pollok and co-workers (Pollok et al. 2015) investigated the effects of tACS over the left primary motor cortex within the alpha and beta-frequency bands on motor sequence learning as indexed by a serial reaction time task (SRTT). Results in 26 participants show that SRTT performance significantly improved at 10 and $20 \mathrm{~Hz}$-tACS. This outcome indicates that tACS facilitates sequence learning and promotes quicker skill acquisition. Nevertheless, as pointed out by Davis et al. (2012), future studies employing tACS need to further clarify the role of beta-frequency neural oscillations in motor control. For example, it would be important to establish how close the stimulating frequency needs to be to the participant's individual beta peak to get the best enhancing effect on performance.

In contrast to tACS, neurofeedback training provides individuals with real-time information about their level of cortical activity via sounds or visual displays. As pointed out by Keizer et al. (2010), with neurofeedback training, brain waves are recorded using electrodes that are likely to pick up signals from theoretically relevant brain areas. An online spectrum analysis is implemented, which enables rewarding participants in order to lead them to increase or decrease the power in the targeted frequency band(s). By doing so, people are able to alter (enhance or reduce) the power of specific frequency bands in their own EEG signal. In this respect, cortical activity may be entrained, changed, or regulated prior to or during physical activity. In one of the first studies on the impact of such EEG-biofeedback procedures on athletic performance, 24 pre-elite archers were examined (Landers et al. 1991).

Two groups were tested based on previous evidence showing that (i) situations requiring attention and preparation elicit slow brain potentials (Rockstroh et al. 1982) and (ii) reduced EEG activity in the left temporal cortex was associated with better sport performance (Hatfield et al. 1984). Therefore, one group received biofeedback designed to augment left temporal low frequency activity (correct biofeedback) and the other group received feedback to augment instead right temporal 
low frequency activity (incorrect biofeedback). The results revealed that the archers who received correct biofeedback compared to incorrect or no biofeedback training significantly improved performance while no effects were observed in selfreported measures of concentration and self-confidence. In another study, effects of real-life neurofeedback training on golf performance were examined by Arns et al. (2008). A personal event-locked EEG profile at frontopolar site (FPz) was calculated for successful versus unsuccessful putts in six amateur golfers. Target frequency bands and amplitudes marking optimal prefrontal brain state were derived from the profile and based on these parameters individual neurofeedback training was applied. The overall percentage of successful putts was significantly larger when feedback was provided as compared to a no-feedback condition. All in all, participants improved their putting performance with neurofeedback related to their personalized EEG profile by an average of $25 \%$. Similarly, in a recent study, Ring and co-workers (2015) examined neurofeedback procedures at frontal site (Fz) in 24 golfers to regulate brain activity towards a reduction of their frontal high-alpha power as a prerequisite prior to successful putting performance. Alpha power was chosen based on previous findings that voluntary self-paced movements (such as golf putts) are preceded by a reduction (i.e., desynchronisation) in EEG alpha power (Leocani et al. 1997). Fz site was relevant because this site was found to capture the strongest differences in high-alpha power between experts and novices and successful and unsuccessful outcomes in the moments preceding golf putts (Cooke et al. 2014). The findings showed that neurofeedback training was successful in reducing the golfers' alpha-band activity while no improvements were found in the putting performance. In a similar study, but without a control group and with a very low sample size, a pre-post intervention of neurofeedback training was conducted to decrease the frontal midline theta amplitude in three highly skilled male golfers (Kao et al. 2014). Low theta-band activity is considered a prerequisite for optimal top-down control and sustained attention. Putting scores and scoring stability improved and the golfers exhibited lower frontal midline theta amplitude during the resting condition following neurofeedback training, pointing out that the tonic reduction of frontal midline theta amplitude may play a role in subsequent performance enhancement. Aside from golf putting studies, beneficial effects of neurofeedback training were shown in rifle shooters as well. Here, expert shooters were asked to maintain intermediate-frequency EEG bandwidths (sensorimotor rhythm and alpha-band) while inhibiting highand low-frequency bandwidths (theta and high beta) for at least $80 \%$ of the practice duration (Rostami et al. 2012). The results revealed improvements of shooting performance when compared to a control group. That is, consolidating lowfrequency brain activity can be useful for improving rifle shooting performance. However, in order to draw valid conclusion on the effect of neurofeedback on sport performance, future studies should start using similar protocols, adequate control conditions, and sample size.

All in all, these results show that neural entrainment through tACS and neurofeedback can speed up the learning of motor skills in sports and boost motor sequence learning to promote optimal performance during competition.

\section{Conclusion}

Noninvasive brain stimulation and neural entrainment have the potential to enhance athletic performance. Even though some studies lack ecological validity towards sports and other studies reported very small sample sizes, it seems plausible that these emerging techniques will be a future way to enhance physical and mental performance in sports. However, it is important to acknowledge that extensive research is needed to verify whether the observed brain stimulation and neural entrainment-induced changes in athletic performance are preserved over time. Previous studies on cognitive functioning have suggested that repetitive sessions of tDCS can increase the effects of stimulation (Nitsche and Paulus 2011), but it remains to be established whether the same applies to athletic performance. Hence, future studies assessing the impact of multiple stimulation sessions and the risk of incurring potential side-effects are necessary. Moreover, optimal protocols of stimulation (e.g., intensity and duration of the stimulation, online vs. offline stimulation, electrode size and number, scalp placement) still need to be identified. Further, in order to find unequivocal evidence that these emerging techniques improve athletic performance, homogeneity across different studies, also in terms of study design and the specific task/ questionnaire used, is mandatory. In addition, by comparing elite to non-elite athletes, future studies need to ascertain whether ceiling effects might prevent any extra improvements. Furthermore, new studies might consider the effect of transcranial random noise stimulation (tRNS) on sport performance. This novel technique requires the application of alternating currents at different frequencies to the scalp and has shown already promising effect on cognition (Cohen Kadosh 2013). Finally, given that many professional athletes gain their prominence through success in "age group" competition (i.e., when they are below the age of 18 in most sports), as proposed by Davis (2014), there is the possibility that a child might take part of the internet-based do-it-yourself movement ("DIYtDCS") or use commercial tDCS devices without the safeguards of the lab or clinic (Steenbergen et al. 2016). As pointed out by Davis (2013), the application of these techniques in sports science raises ethical concerns about neurodoping in sports. Notably, brain stimulation and neural entrainment may be considered artificial supplements to normal training in sports, provoking ethical debates about their application. In 
accordance with Davis (2013), if used according to safety protocols, we believe that neuro-enhancement during training should not be considered unethical and illicit in sport. Although more research is needed to fully understand the effects of noninvasive brain stimulation and neural entrainment on athletic performance, we conclude that these emerging techniques are promising tools to enhance athletic performance such as motor learning, muscular strength, increased learning rate of specific motor skills, sleep, and fatigue.

Open Access This article is distributed under the terms of the Creative Commons Attribution 4.0 International License (http:// creativecommons.org/licenses/by/4.0/), which permits unrestricted use, distribution, and reproduction in any medium, provided you give appropriate credit to the original author(s) and the source, provide a link to the Creative Commons license, and indicate if changes were made.

\section{References}

Antal, A., Nitsche, M. A., Kincses, T. Z., Kruse, W., Hoffmann, K. P., \& Paulus, W. (2004). Facilitation of visuo-motor learning by transcranial direct current stimulation of the motor and extrastriate visual areas in humans. European Journal of Neuroscience, 19(10), 2888 2892.

Antal, A., Nitsche, M. A., Kruse, W., Kincses, T. Z., Hoffmann, K. P., \& Paulus, W. (2004). Direct current stimulation over V5 enhances visuomotor coordination by improving motion perception in humans. Journal of Cognitive Neuroscience, 16(4), 521-527.

Antal, A., Boros, K., Poreisz, C., Chaieb, L., Terney, D., \& Paulus, W. (2008). Comparatively weak after-effects of transcranial alternating current stimulation (tACS) on cortical excitability in humans. Brain Stimulation, 1(2), 97-105.

Arns, M., Kleinnijenhuis, M., Fallahpour, K., \& Breteler, R. (2008). Golf performance enhancement and real-life neurofeedback training using personalized event-locked EEG profiles. Journal of Neurotherapy, 11(4), 11-18.

Chaieb, L., Antal, A., \& Paulus, W. (2011). Transcranial alternating current stimulation in the low $\mathrm{kHz}$ range increases motor cortex excitability. Restorative Neurology and Neuroscience, 29(3), 167-175. doi:10.3233/RNN-2011-0589.

Cogiamanian, F., Marceglia, S., Ardolino, G., Barbieri, S., \& Priori, A. (2007). Improved isometric force endurance after transcranial direct current stimulation over the human motor cortical areas. European Journal of Neuroscience, 26(1), 242-249.

Cohen Kadosh, R. (2013). Using transcranial electrical stimulation to enhance cognitive functions in the typical and atypical brain. Translational Neuroscience, 4(1), 20-33.

Cooke, A., Kavussanu, M., Gallicchio, G., Willoughby, A., McIntyre, D., \& Ring, C. (2014). Preparation for action: psychophysiological activity preceding a motor skill as a function of expertise, performance outcome, and psychological pressure. Psychophysiology, 51(4), 374-384.

Davis, N. J. (2013). Neurodoping: brain stimulation as a performanceenhancing measure. Sports Medicine, 43(8), 649-653.

Davis, N. J. (2014). Transcranial stimulation of the developing brain: a plea for extreme caution. Frontiers in Human Neuroscience, 8, 600.

Davis, N. J., Tomlinson, S. P., \& Morgan, H. M. (2012). The role of betafrequency neural oscillations in motor control. The Journal of Neuroscience, 32(2), 403-404.

Furuya, S., Nitsche, M. A., Paulus, W., \& Altenmüller, E. (2013). Early optimization in finger dexterity of skilled pianists: implication of transcranial stimulation. BMC Neuroscience, 14(1), 1.
Grosprêtre, S., Ruffino, C., \& Lebon, F. (2016). Motor imagery and cortico-spinal excitability: a review. European Journal of Sport Science, 16(3), 317-324.

Hatfield, B. D., Landers, D. M., \& Ray, W. J. (1984). Cognitive processes during self-paced motor performance: an electroencephalographic profile of skilled marksmen. Journal of Sport Psychology, 6(1), $42-59$.

Kao, S. C., Huang, C. J., \& Hung, T. M. (2014). Neurofeedback training reduces frontal midline theta and improves putting performance in expert golfers. Journal of Applied Sport Psychology, 26(3), 271286.

Keizer, A. W., Verschoor, M., Verment, R., \& Hommel, B. (2010). The effect of gamma enhancing neurofeedback on measures of featurebinding flexibility and intelligence. International Journal of Psychophysiology, 75, 25-32.

Landers, D. M., Petruzzello, S. J., Salazar, W., Crews, D. J., Kubitz, K. A., Gannon, T. L., et al. (1991). The influence of electrocortical biofeedback on performance in pre-elite archers. Medicine \& Science in Sports \& Exercise, 23(1), 123-129.

Leocani, L., Toro, C., Manganotti, P., Zhuang, P., \& Hallett, M. (1997). Event-related coherence and event-related desynchronization/ synchronization in the $10 \mathrm{~Hz}$ and $20 \mathrm{~Hz}$ EEG during self-paced movements. Electroencephalography and Clinical Neurophysiology/Evoked Potentials Section, 104(3), 199-206.

Moliadze, V., Antal, A., \& Paulus, W. (2010). Boosting brain excitability by transcranial high frequency stimulation in the ripple range. Journal of Physiology, 588(24), 4891-4904. doi:10.1113/jphysiol.2010.196998.

Moliadze, V., Atalay, D., Antal, A., \& Paulus, W. (2012). Close to threshold transcranial electrical stimulation preferentially activates inhibitory networks before switching to excitation with higher intensities. Brain Stimulation, 5(4), 505-511.

Montenegro, R. A., Farinatti, P. D. T. V., Fontes, E. B., da Silva Soares, P. P., da Cunha, F. A., Gurgel, J. L., ..., Okano, A. H. (2011). Transcranial direct current stimulation influences the cardiac autonomic nervous control. Neuroscience Letters, 497(1), 32-36.

Nitsche, M. A., \& Paulus, W. (2000). Excitability changes induced in the human motor cortex by weak transcranial direct current stimulation. The Journal of Physiology, 527(3), 633-639.

Nitsche, M. A., \& Paulus, W. (2011). Transcranial direct current stimulation-update 2011. Restorative Neurology and Neuroscience, 29, 463-492.

Nitsche, M. A., Liebetanz, D., Antal, A., Lang, N., Tergau, F., \& Paulus, W. (2003). Modulation of cortical excitability by weak direct current stimulation-technical, safety and functional aspects. Supplements to Clinical Neurophysiology, 56(3), 255-276.

Nitsche, M. A., Schauenburg, A., Lang, N., Liebetanz, D., Exner, C., Paulus, W., et al. (2003). Facilitation of implicit motor learning by weak transcranial direct current stimulation of the primary motor cortex in the human. Journal of Cognitive Neuroscience, 15(4), 619-626.

Nitsche, M. A., Cohen, L. G., Wassermann, E. M., Priori, A., Lang, N., Antal, A., ..., Pascual-Leone, A. (2008). Transcranial direct current stimulation: state of the art 2008. Brain Stimulation, 1(3), 206-223.

Okano, A. H., Fontes, E. B., Montenegro, R. A., Farinatti, P. D. T. V., Cyrino, E. S., Li, L. M., .. \& Noakes, T. D. (2013). Brain stimulation modulates the autonomic nervous system, rating of perceived exertion and performance during maximal exercise. British Journal of Sports Medicine, bjsports-2012.

Pollok, B., Boysen, A. C., \& Krause, V. (2015). The effect of transcranial alternating current stimulation (tACS) at alpha and beta frequency on motor learning. Behavioural Brain Research, 293, 234-240.

Reato, D., Rahman, A., Bikson, M., \& Parra, L. C. (2010). Low-intensity electrical stimulation affects network dynamics by modulating population rate and spike timing. The Journal of Neuroscience, 30(45), 15067-15079. doi:10.1523/JNEUROSCI.2059-10.2010. 
Reato, D., Rahman, A., Bikson, M., \& Parra, L. C. (2013). Effects of weak transcranial alternating current stimulation on brain activitya review of known mechanisms. Frontiers in Human Neuroscience, 7, 1-8. doi:10.3389/fnhum.2013.00687.

Reis, J., Schambra, H. M., Cohen, L. G., Buch, E. R., Fritsch, B., Zarahn, E., et al. (2009). Noninvasive cortical stimulation enhances motor skill acquisition over multiple days through an effect on consolidation. Proceedings of the National Academy of Sciences, 106(5), 1590-1595.

Ring, C., Cooke, A., Kavussanu, M., McIntyre, D., \& Masters, R. (2015). Investigating the efficacy of neurofeedback training for expediting expertise and excellence in sport. Psychology of Sport and Exercise, $16,118-127$.

Rockstroh, B., Elbert, N., Birbaumer, N., \& Lutzenberger, W. (1982). Slow brain potentials and behavior. Baltimore: Urban \& Schwarzenberg.

Rostami, R., Sadeghi, H., Karami, K. A., Abadi, M. N., \& Salamati, P. (2012). The effects of neurofeedback on the improvement of rifle shooters' performance. Journal of Neurotherapy, 16(4), 264-269.
Steenbergen, L., Sellaro, R., Hommel, B., Kühn, S., \& Colzato, L. S. (2016). "Unfocus" on foc.us: commercial tDCS headset impairs working memory. Experimental Brain Research, 234, 637-643.

Vitor-Costa, M., Okuno, N. M., Bortolotti, H., Bertollo, M., Boggio, P. S., Fregni, F., et al. (2015). Improving cycling performance: transcranial direct current stimulation increases time to exhaustion in cycling. PloS One, 10(12), e0144916.

Williams, P. S., Hoffman, R. L., \& Clark, B. C. (2013). Preliminary evidence that anodal transcranial direct current stimulation enhances time to task failure of a sustained submaximal contraction. PloS One, 8(12), e81418.

Zhu, F. F., Yeung, A. Y., Poolton, J. M., Lee, T. M., Leung, G. K., \& Masters, R. S. (2015). Cathodal transcranial direct current stimulation over left dorsolateral prefrontal cortex area promotes implicit motor learning in a golf putting task. Brain Stimulation, 8(4), 784786. 